Z Gerontol Geriat 2018 $51: 573-578$

https://doi.org/10.1007/s00391-017-1230-6

Eingegangen: 30. Januar 2016

Überarbeitet: 7. Januar 2017

Angenommen: 27. März 2017

Online publiziert: 18. April 2017

(c) Der/die Autor(en) 2017

CrossMark

W. Weinrebe ${ }^{1,2}(\mathbb{D}) \cdot A$. Moutaouakil' $\cdot$ K. Risz' $\cdot$ M. Martin' $\cdot$ K. Jeckelmann' $\cdot$ S. Goetz

'Geriatric Medicine and Rehabilitation, Hopital Fribourgois, Meyriez, Schweiz

${ }^{2}$ University of Witten Herdecke, Witten-Herdecke, Germany

\title{
89 Jahre, Depression, Sturz mit Beckenfraktur, schwerste Verwirrtheit - das serotonerge Syndrom
}

\section{Differenzialdiagnose, Bedeutung von CYP450 und ökonomische Betrachtungen}

grad M4). Die perianale Sensibilität und der Sphinktertonus waren erhalten. Der übrige Status war unauffällig. In der radiologischen Abklärung der LWS (Lendenwirbelsäule) konnte eine frische Fraktur ausgeschlossen werden. Es zeigten sich eine mäßige bis deutliche Spondylarthrose und eine konzentrische Koxarthrose rechts bei leichtgradig erhöhtem CRP (40 mg/l). Frau K. wurde zur analgetischen Therapie und zur Mobilisierung unter Anleitung der Physiotherapie hospitalisiert. Die bei Eintritt festgestellte Hypokaliämie wurde mit Kalium und Magnesium substituiert. Während des Klinikaufenthalts stürzte Frau K. am 24.12.2015 abends aus ca. $50 \mathrm{~cm}$ Höhe auf den Boden. Sie zog sich dabei eine untere Schambeinastfraktur links und eine Acetabulumfraktur links zu.

Die Schmerzmedikation musste angepasst werden, da Frau K. über sehr starke immobilisierende Schmerzen im linken Becken klagte. Die Schmerzmedikation mit Oxycodon (Oxycontin) wurde erweitert und bei ungenügendem Ansprechen auf einen kontinuierlichen Schmerzperfusor mit Pethidin (Pethidin) und Novaminsulfon (Novalgin) umgestellt. Zusätzlich erhielt Frau K. ergänzend Paracetamol (Dafalgan) i.v. und Tolperison (Mydocalm) p.o. Eine einfache Zystitis, im Urinstatus nitritpositiv und im Uricult E.-coli-sensibel aufalle gängigen Antibiotika, wurde antibiotisch mit einer einma- ligen Gabe von $3 \mathrm{~g}$ Fosfomycin (Monuril) behandelt.

Im Verlauf stellte sich nach der vorliegenden Dokumentation ( $\bullet$ Tab. 1) ein Delir ein, das sich mit Desorientiertheit, Halluzinationen (die Patientin führte Selbstgespräche), psychomotorischer Unruhe wie Nesteln und teilweiser Somnolenz präsentierte. Die Symptome waren v. a. nachts stark ausgeprägt, zusätzlich zu Angstzuständen. Eine neuroleptische Therapie mit Quetiapin, diekontinuierlich gesteigert wurde, brachte eine gewisse Besserung. So war Frau K. teilweise wieder imstande, Angehörige bei ihren Namen zu nennen. Das am 28.12.2015 durchgeführte CT (Computertomographie) vom Becken zeigte neben der vorderen Acetabulumfraktur links eine Dislokation der unteren Schambeinastfraktur links und eine zusätzliche Fraktur des Sakrums ohne Beteiligung der Neuroforamina (•Abb. 1). Wegen der komplexen Situation wurde Frau K. zur Weiterbehandlung auf die akutgeriatrische Abteilung überwiesen.

Bei geriatrischer Übernahme der Patientin um 10 Uhr morgens stand nach konservativ versorgter Beckenringfraktur und angegebener „demenzieller Entwicklung" formal die „delirante Symptomatik“ im Vordergrund. Die Symptomatik hatte nach der vorliegenden klinischen Dokumentation spontan am Tag 1 nach stationärer Aufnahme begonnen. und Großzehenextension rechts (Kraft- 


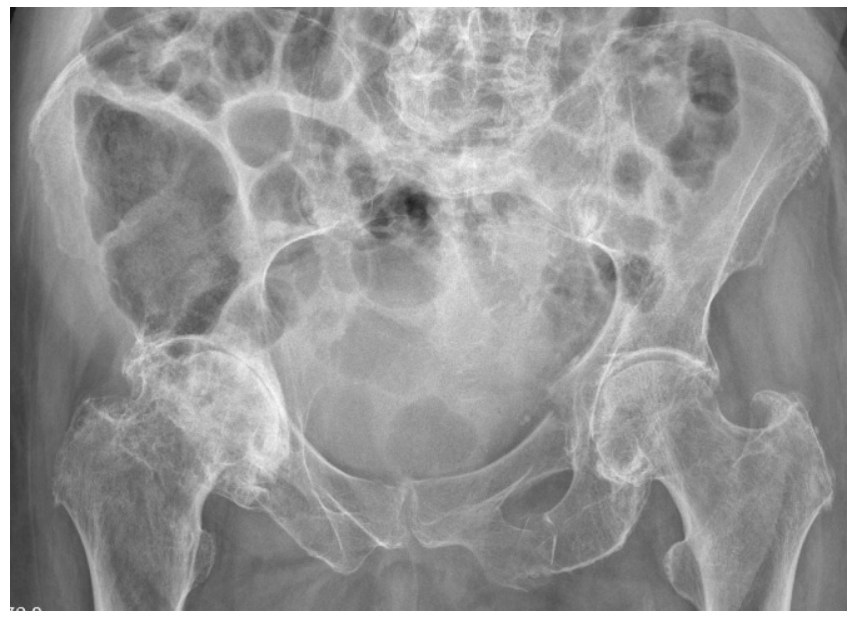

Abb. $1<$ Schwere Koxarthrose rechts, vordere Acetabulumfrakturlinks, Dislokation der unteren Schambeinastfraktur links, Fraktur des Sakrums

\section{A. Genetische Ergebnisse}

\begin{tabular}{l|ll}
\hline \multirow{4}{*}{ Zytochrome } & \multicolumn{1}{c}{ Gen } & \multicolumn{1}{c}{ Genotyp } \\
\cline { 2 - 3 } & CYP1A2 & $* 1 \mathrm{~A} /{ }^{*} 1 \mathrm{~F}$, Heterozygot \\
\cline { 2 - 3 } & CYP2D6 & $* 4 /{ }^{*} 4$, Homozygot \\
\cline { 2 - 3 } & CYP2C19 & ${ }^{*} 1 /{ }^{*} 1$, keine Variation \\
\cline { 2 - 3 } & CYP2B6 & ${ }^{*} 1 /{ }^{*} 22$, Heterozygot \\
\cline { 2 - 3 } & CYP3A4 & ${ }^{*} 1 /{ }^{*} 1$, keine Variation \\
\cline { 2 - 3 } & CYP3A5 & $* 3 / * 3$, Homozygot
\end{tabular}

Abb. $2 \Delta$ Ergebnisse der pharmakogenetischen Analyse

Die Symptome waren fluktuierend über den Tag und die Nacht. Die Patientin zeigte eine Unfähigkeit, dem Gespräch oder dem Gesagtem zu folgen, und das Denken war desorganisiert. Intermittierend schien sie schläfrig zu sein und war nichtweckbar. Die CAM-Score-Kriterien für ein Delir waren erfüllt [1]. Zur Beurteilung der Delirschwere wurde der CAM-S durchgeführt. Der CAM-S zeigte mit 6 Punkten ein schweres Delir [2]. Formal handelte es sich um ein schweres, hypermotorisches Delir.

Klinisch-neurologisch war die Patientin auffallend schreckhaft und ängstlich, zeigte sich motorisch sehr unruhig und zittrig. Bei nur leichtem Beklopfen oder Berühren der Arme wurden spontan Reflexe ausgelöst. Die Beine wurden ständig bewegt. Außerdem konnte sie nicht mehr sprechen. Die ausgesprochenen Worte waren unverständlich und bruchhaft.

Es bestand ein Bulbuswandern bei normal weiten Pupillen. Auffällig waren zudem ein insgesamt erhöhter Tonus über allen Extremitäten und ein relativ steifer Nacken. Differentialdiagnostisch standen eine Meningitis und ein zerebrales, ischämisches oder embolisches Ereignis im Raum. Die Nackensteifigkeit war jedoch nicht sehr ausgeprägt, es bestanden weder Kopfschmerzen, Kernigoder Brudzinksi-Zeichen und auch keine Lichtscheu. Für ein Insultereignis zeigten sich keinerlei Foki im Sinne einer Parese oder einer motorischen Schwäche. Alle Extremitäten wurden spontan und kräftig bewegt. Die Bewegungen waren allerdings unkoordiniert; die Sprache war nicht verständlich. Ein CCT war aufgrund der Unruhe der Patientin nur sehr schwierig durchzuführen, zeigte aber keinen pathologischen Fokus. Eine Lumbalpunktion und ein MRT waren in der jetzigen Situation nicht durchführbar. Das Thoraxröntgenbild war unauffällig. Klinisch bestanden eine Tachykardie und Hypertonie; sie entwickelte Fieber und Durchfall. Laborchemisch bestanden eine Elektrolytentgleisung und eine Infektkonstellation mit CRP-Erhöhung auf $97 \mathrm{mg} / \mathrm{dl}$; die Leukozyten lagen bei
10.200. Die BGA zeigte bei Tachypnoe, Tachykardie und septischen Temperaturen das Bild einer nichtkompensierten respiratorischen Alkalose.

Zunächst wurden die Symptome, mit denen des serotonergen Syndroms nach Sternberg [3-6] verglichen: autonome Instabilität, neuromuskuläre Zeichen, Veränderungen der Kognition und des Verhaltens in der Gegenwart von serotonerger Medikation. Dabei zeigte sich, dass die Symptome der Patientin deutlich mehr als die geforderten 3 Bereiche nach Sternberg abdeckten. Auch die „Hunter decision rules“ $[7,8]$ für das serotonerge Syndrom waren positiv. Sie müssen mindestens in einem Bereich positiv sein - bei der Patientin waren es 3 Bereiche [2, 4, 5].

\section{Diagnosen}

- Dringender V.a. mittelschweres bis schweres serotonerges Syndrom,

- hypermotorisches Delir,

- möglicherweise arthritischer Schub bei einer konzentrischen Koxarthrose rechts,

- prim. chronisches Schmerzsyndrom im rechten Bein und Lokomotionsstörungen,

- multifaktorieller Sturz (Tolperison, Delir, Immobilität),

- sekundär akutes schwerstes Schmerzsyndrom bei Beckenringfraktur,

- arterielle Hypertonie.

Nach Übernahme der Patientin in die Akutgeriatrie wurde zunächst die Schmerzmedikation mit Pethidin beendet und auf ein niedrig dosiertes Fentanyl (Durogesic Matrix) (12,5 $\mu \mathrm{g}$ ) umgesetzt, da nach Studienlage Fentanyl als Schmerzmedikament weniger bis selten serotonerge Syndrome auslöst oder unterhält $[7,8]$. Alle zentral wirkenden Medikamente, insbesondere Mirtazapin (Mirtazapin), Citalopram (Cipramil), Quetiapinum (Quetiapin) und Lorazepanum (Lorazepam) und das Tolperison (Mydocalm) wurden gestoppt. Es wurden $5 \mathrm{mg}$ Diazepanum (Diazepam) rektal verabreicht (mehrfach täglich nach Bedarf). Bei ansteigender und septischer Temperatur bis auf $39,4^{\circ}$ Temperatur wurden Blutkulturen ent- 
nommen und physikalische Kühlungsmaßnahmen durchgeführt. Cefepimum (Cefepim) wurde nierendosisangepasst 2-mal 500mg i.v. verabreicht. Bei ansteigenden Retentionsparametern wurde angepasst i.v. Volumen ersetzt, und die Elektrolyte wurden ausgeglichen (Hypernatriämie $(156 \mathrm{mmol} / \mathrm{l})$, (Hypokaliämie $(3 \mathrm{mmol} / \mathrm{l})$. Es wurde ein mehrfach tägliches Monitoring der Symptome festgelegt.

Die Patientin wurde 24 h nach Absetzen der Medikation etwas ruhiger. Sie entfieberte nach $48 \mathrm{~h}$, wurde erstmals wacher und öffnete gezielt die Augen. Sie entwickelte aber starke Entzündungsparameter bei weiter unklarem Fokus (Blutkulturen blieben negativ) und sie wurde breit antibiotisch abgedeckt. Achtzig Stunden nach Absetzen war das serotonerge Syndrom fast komplett behoben; sie nestelte noch etwas, aber die neurologischen Symptome waren nahezu verschwunden. Sie konnte wieder langsam Nahrung zu sich nehmen, nachdem sie zuvor periphervenös ernährt worden war (SMOF Kabiven peripher). Die Patientin konnte wieder reden und Kontakt aufnehmen. Sie war orientiert, konnte sich aber nicht mehr erinnern. Zu diesem Zeitpunkt trat das depressive Syndrom wieder zutage, das im Vorfeld mit Mirtazapin und Citalopram anbehandelt worden war. Eine Wiederaufnahme der Medikation wurde jedoch noch nicht durchgeführt, da es in der Literatur dazu negative Informationen gab [9] und ein Wiederauftreten des serotonergen Syndroms unbedingt vermieden werden sollte. Unter der Schmerztherapie war auch eine Mobilisation in den Rollstuhl möglich. Eine Neuorganisation der Versorgung in einem Pflegebett wurde geplant, da sie mindestens noch 6 Wochen mit der Beckenfraktur nicht belastbar sein würde. Von dort sollte dann die weitere Versorgung final geplant werden.

Differenzialdiagnostisch [10-12] war auch ein Hyperthermiesyndrom zu diskutieren, da die Gabe von Tolperison dieses auslösen kann, zumal es in Maximaldosis gegeben wurde und gleichzeitig mehrere Medikamente eine CYP2D6Affinität hatten. Bei der Patientin war jedoch nicht bekannt, welche Form von „metabolizer“ sie war. Eine Bestimmung

Z Gerontol Geriat 2018 · 51:573-578 https://doi.org/10.1007/s00391-017-1230-6

(c) Der/die Autor(en) 2017

W. Weinrebe · A. Moutaouakil · K. Risz · M. Martin · K. Jeckelmann · S. Goetz

89 Jahre, Depression, Sturz mit Beckenfraktur, schwerste Verwirrtheit - das serotonerge Syndrom. Differenzialdiagnose, Bedeutung von CYP450 und ökonomische Betrachtungen

\section{Zusammenfassung}

Es wird eine Fallkasuistik einer 89-jährigen Patientin geschildert, die wegen einer Immobilität bei Schmerzen in die Klinik eingewiesen wurde. Nachdem zunächst die Ursache der Schmerzen klinisch geklärt und therapeutisch versorgt wird, zeigt sich im weiteren klinischen Verlauf bei dieser hochbetagten Frau ein gefährlicher Symptomkomplex aus Agitiertheit, Verwirrtheit und kognitivem Abbau, der gravierende Formen annimmt. Die Aufarbeitung dieses Falls zeigt einerseits eine typische Konstellation von Schmerz und Depression im Alter; andererseits ist die medikamentöse Behandlung in diesem Fall nicht untypisch und konnte ein schweres serotonerges Syndrom entwickeln. Interaktion, Diagnosefindung, Differenzialdiagnose, pharmakologische, funktionelle, kodiertechnische und ökonomische Aspekte des Verlaufs werden diskutiert.

Schlüsselwörter

Medikamenteninteraktionen - CYP450 .

Geriatrischer Patient · Depression .

Schmerztherapie

\section{Age 89 years, depression, fall with pelvic fracture, severe confusion - serotonin syndrome. Differential diagnosis, importance of CYP450 and economic considerations}

\section{Abstract}

A case study of an 89-year-old patient is reported, who was admitted to hospital because of immobility due to pain. After the cause of the pain could initially be clarified and treated, the further clinical course in this very old woman showed an alarming symptom complex of agitation, confusion and cognitive deterioration, which took on grave forms. The work-up of this case showed a typical constellation of pain and depression in old age; however, the pharmaceutical treatment in this case is not atypical and could lead to a severe serotonin syndrome. The interaction, diagnostics, differential diagnosis, pharmacological, functional, codification and economic aspects of the course of the disease are discussed.

Keywords

Medical side effects - Geriatric patients .

Depression · Pain therapy wurde erst postklinisch durchgeführt (- Abb. 2).

Das anticholinerge Syndrom entfiel, da keine Verwendung von trizyklischen Antidepressiva oder andere anticholinerg wirkenden Substanzen verwendet wurden. Das maligne neuroleptische Syndrom entfiel, denn es erfolgte zwar eine Gabe von Quetiapin und Haloperidol, aber die Symptomatik zeigte keine Hyporeflexie, Koma oder Katatonie.

Die medikamentöse Anamnese und die pharmakologische Eigenschaften (inklusive der Eliminationshalbwertzeit der verabreichten Medikationen) [13, 14] brachte zutage, dass eine serotonerge Risikokonstellation vorlag: Die Patientin erhielt wegen des ausgepräg- ten Schmerzsyndroms bei komplexer Beckenringfraktur eine kombinierte i. v.-Schmerzmedikation mit Pethidin $400 \mathrm{mg}+2,5 \mathrm{~g}$ Novaminsulfon mit 2-4 ml/h. Die Gesamtmenge war damit in $24 \mathrm{~h} 400-800 \mathrm{mg}$ Pethidin und 2,5-5 g Novaminsulfon i. v. Parallel dazu war die Medikation der Patientin mit Mirtazapin, Citalopram und Quetiapin und Lorazepam weitergeführt worden.

Es ist pathophysiologisch nachgewiesen [15], dass eine längerfristige Gabe von Pethidin mit der Kumulation seines aktiven Metaboliten Norpethidin zu einer Hemmung der Wiederaufnahme von Serotonin aus dem synaptischen Spalt führt.

Auch Citalopram wirkt als selektiver Serotonin-Wiederaufnahmehem- 


\section{Übersichten}

\begin{tabular}{|c|c|c|}
\hline to & um 20.38 & Aufnahme, orientiert, klar, schmerzgeplagt, teilmobil \\
\hline $\mathrm{t} 1$ & um 22.12 & Delirentwicklung mit Unruhe, Verwirrtheit \\
\hline \multirow[t]{3}{*}{$\mathrm{t} 2$} & um 03.45 & Tag-Nacht-Umkehr, verwirrt, hypermotorisches Delir \\
\hline & um 19.00 & Sturz auf dem Weg zur Toilette, Beckenringfraktur \\
\hline & um 21.00 & $\begin{array}{l}\text { Beginn der Perfusortherapie mit Pethidin + Novalgin, Patientin schläft } \\
\text { tief }\end{array}$ \\
\hline \multirow[t]{2}{*}{ t3 } & um 13.19 & Allgemeinzustand schlechter, trinkt nicht mehr, reagiert kaum \\
\hline & um 14.03 & Kaltschweißige Patientin \\
\hline \multirow[t]{2}{*}{ t4 } & um 10.07 & Unruhe zunehmend, kaum zu beruhigen \\
\hline & um 23.12 & Weitere motorische Unruhe, fahrig, zittrig \\
\hline t5 & um 15.00 & $\begin{array}{l}\text { Kann keine Anforderungen mehr umsetzen, Becher fällt aus der Hand, } \\
\text { greift in die Luft, nestelt }\end{array}$ \\
\hline t6 & um 04.30 & Schreit, schlägt um sich, keine Kontaktaufnahme mehr möglich \\
\hline \multirow[t]{2}{*}{ t7 } & um 04.35 & Ataktische Zuckungen, zittert am ganzen Körper, Tachykardie, Rigor \\
\hline & um 10.00 & Verlegung von der Orthopädie in die Akutgeriatrie \\
\hline
\end{tabular}

mer. Diese beiden Substanzen führten zu einem deutlich erhöhten Serotoninangebot im synaptischen Spalt. Zudem kann Oxycodon über den Anstieg von Serotoninfreisetzung in weiten Bereichen des Vorderhirns die Konzentration steigern und dann zusammen mit Citalopram ein serotonerges Syndrom auslösen. Weitere Kombinationen mit serotonergen Effekten sind möglich [16].

\section{Ökonomische Betrachtung des serotonergen Syndroms in den DRG}

Da das serotonerge Syndrom nicht kodierbar ist, war es schwierig, die dahinterliegenden Aufwände adäquat abzubilden: Personalaufwand (durchgehende, hochfrequente Versorgung mit $2 \mathrm{MA}$ des Pflegepersonals, 3 tägliche ärztliche Visiten, stärkere Einbindung von leitenden Ärzten, multiprofessionelle Versorgung, mehrere Disziplinen mit Anästhesisten, Orthopäden, Internisten, Geriatern, Ernährungsmediziner), medizinische Diagnostik (hochfrequente Laborkontrollen, teure Diagnostik [Prokalzitonin, Gerinnung, Schädel-CT]), medizinische Therapie (i. v.-Schmerzperfusor, i.v.-Antibiotikagabe, parenterale Ernährung, multiprofessionelle Therapiekonzepte mit Physiotherapie, Ergotherapie, aktivierend therapeutische Pflege, Sozialarbeit, Neuropsychologie).

Man könnte die entstandenen Kosten durch eine Vergleichsberechnung ab- schätzen, denn es besteht klinisch eine Nähe zum hypermotorischen Delir, bzw. Teile des serotonergen Syndroms sind klinisch so klassifizierbar. Die Kostenstruktur des hypermotorischen Delirs wurde publiziert [17]. Hypermotorische Delirien dauern im Schnitt 1,4 Tage und kosten ca. $1000 €$ für Personal und Medikation bei deutschen Personal- und Sachkosten. Bildet man modellhaft klinisch das serotonerge Syndrom als hypermotorisches Delir (hier Dauer über 9 Tage) $a b$, sind die Kosten abschätzbar: Daraus ergibt sich ein geschätzter Kostenfaktor von $6400 € / 19.200 \mathrm{sFr}$. für dieses serotonerge Syndrom. Hinzukämen allerdings noch die Kosten für Medikamente (i.v.Schmerztherapie und Antibiose) und labortechnische (Prokalzitonin, Blutkulturen) und radiologische Zusatzdiagnostik (CT-Schädel), denn sie sind deutlich höher als beim Delirpatienten.

Die modellhafte Rechnung (• Tab. 2) zeigt ökonomisch, dass die Aufwände für ein serotonerges Syndrom als Kosten nicht im DRG-System gedeckt sind und letztlich die Mehrkosten von der Klinik getragen werden müssen. Sie zeigt aber auch, dass die in den DRG angelegte interdisziplinäre Zusammenarbeit zwischen operativen und geriatrischen Abteilungen einen optimierten Verlauf und auch eine Kostendeckung dieser enormen $\mathrm{Zu}$ satzkosten ermöglichen kann. Besonders deutlich ist dieser Effekt, wenn die Patientin frühestmöglich in die Akutgeriatrie gesteuert worden wäre.

\section{Diskussion}

Das serotonerge Syndrom ist nicht selten; eine genaue Inzidenz ist aber nicht bekannt, da viele Ereignisse unerkannt verlaufen. Bei der Gabe von SSRI stellen sich in 16-18 \% der Fälle Nebenwirkungen oder toxische Auswirkungen ein. In einer Statistik des U.S. Poison Control Centers aus den Jahren 2002 und 2005 waren unter den gemeldeten Vergiftungen mit SSRI 0,2\% Todesfälle beschrieben worden [18-20]. Antidepressiva sind die mit Abstand am häufigsten verordneten Substanzen. Der am häufigsten verschriebene selektive „serotonin reuptake inhibitor" (SSRI) ist Citalopram, dessen "defined daily doses“ (DDD) lag 2011 bei 338,7 Mio. Mirtazapin ist nach Citalopram das Psychopharmakon mit den zweithäufigsten Verordnungen mit einer DDD von 150,4 Mio. im Jahr 2011 [21].

Therapierende und verordnende Ärzte müssen sich die Zusammenhänge dieser Substanzen und ihrer Gefahren klarmachen. Dies gilt insbesondere für die multimorbiden, geriatrischen Patienten, die häufig mehr als 6 Medikamente und sehr oft Antidepressiva verschrieben bekommen. Aber auch andere Antidepressiva, wie Sertralin oder Fluvoxamin, können zur Induktion des serotonergen Syndroms führen $[22,23]$.

In dieser hochbetagten, geriatrischen Patientengruppe sind Schmerzen ein häufiges Thema. Der Arzneimittelreport 2012 [24] zeigt, dass die Schmerzmedikamente inzwischen auf Platz 2 aller Verschreibungen liegen. Damit ist auch die Wahrscheinlichkeit, bei Schmerzen ein Opioid verschrieben zu bekommen und darauffolgend ein serotonerges Syndrom zu erleben, deutlich erhöht [25]. Bei der Patientin waren die rechtsseitig geklagten Schmerzen des Beins und das inflammatorische Syndrom möglicherweise den schweren degenerativen Veränderungen am rechten Hüftgelenk geschuldet.

Die Ursache des Sturzes ist multimodal bedingt. Ein wichtiger Punkt ist möglicherweise die Tatsache, dass die Patientin bei Aufnahme Tolperison (Mydocalm) erhalten hat, ein zentral wirkendes Muskelrelaxans. Die Dosis war zwar mit 450 mg korrekt, aber die Substanz 


\begin{tabular}{|c|c|c|c|c|c|c|c|}
\hline DRG & DRG & CMP & Erlös & VD & Tagessatz & Kosten & Ertrag \\
\hline $\begin{array}{l}\text { G-DRG (LBFW } \\
3232,73 €)\end{array}$ & L63F & 1,26 & 4073,23 & 21 & 193 & 6400 & -2326 \\
\hline Mit 8-550.1 & L44Z & 2,275 & 7345,46 & 21 & 350 & 6400 & +945 \\
\hline $\begin{array}{l}\text { Mit 8-550.1 } \\
\text { Sofort in Geriatrie }\end{array}$ & L44Z & 2,275 & $\begin{array}{l}\delta^{\mathrm{a}} \\
+1400 €\end{array}$ & 17 & 423 & 6400 & +2345 \\
\hline $\begin{array}{l}\text { Swiss-DRG (Fallwert } \\
10.000 \text { sFr.) }\end{array}$ & L63B & 1,2 & 12.000 & 21 & 571 & 19.200 & -6800 \\
\hline Mit 93.89.91 & A95C & 2,305 & 23.050 & 21 & 1097 & 19.200 & +3850 \\
\hline $\begin{array}{l}\text { Mit } 93.89 .91 \\
\text { Sofort in Geriatrie }\end{array}$ & A95C & 2,305 & $\begin{array}{l}\delta^{b} \\
+6120 \mathrm{sFr}\end{array}$ & 17 & 1355 & 19.200 & +9970 \\
\hline \multicolumn{8}{|c|}{$\begin{array}{l}\text { a } \delta \text {-Ertrag von }+1400 € \text { errechnet sich aus: Verlust an DRG-Erträgen bei } 0,7 \text { CMP und } 4 \text { Tagen Behand- } \\
\text { lung }(1200 €)+\text { Verweildauereffekt }-4 \text { Tage }(200 €) \text { zwischen } 21 \text { und } 17 \text { Tage Behandlung) } \\
\text { b } \delta \text {-Ertrag von }+6,120 \text { sFr. errechnet sich aus: Verlust an DRG-Erträgen bei 0,7 CMP und } 4 \text { Tagen Be- } \\
\text { handlung ([4000 sFr.] + Verweildauereffekt -4 Tage [2120 sFr.] zwischen } 21 \text { und } 17 \text { Tage Behandlung) } \\
\text { LBFW Landesbasisfallwert }\end{array}$} \\
\hline
\end{tabular}

wird ebenso über CYP2D6 verstoffwechselt, wie das Oxycodon und das Mirtazepin. CYP2D6 ist am Stoffwechsel etwa jedes 4 . Arzneimittels beteiligt, darunter vieler Antidepressiva, Neuroleptika, $\beta$-Adrenozeptor-Antagonisten, Antiarrhythmika, Antitussiva und Antiemetika. Eine genetisch bedingte Defizienz führt zu einer deutlich verlangsamten Elimination aus dem Körper, was zu einer relativen Überdosierung mit den entsprechend verstärkten Nebenwirkungen führt. CYP2D6 oxidiertbzw. hydroxyliert in der Leber bestimmte Substrate (Pharmaka) und aktiviert (Pro-Drug) oder deaktiviert diese dadurch. Das CYP2D6 kann als „poor metabolizer“" oder als ,ultra extensive metabolizer" vorliegen - im ersten Fall würde sich die Wirkung der Medikamente potenzieren. Die Bestimmung der CYP2D6-Aktivität bei der Patientin bestätigte, dass sie ein „poor metabolizer" war, was die Auslösung des serotonergen Syndroms pharmakologisch erklärt. So ist es möglich, dass auch $450 \mathrm{mg}$ eine Dosis ergeben und trotz der kurzen HWZ mit für den Sturz, die Unruhe, den Tremor, das Schwitzen und die Temperatur verantwortlich sein können [26]. Ein weiterer Risikofaktor hinsichtlich der Sturzgenese ist das Delir, das sich schon kurz nach der Aufnahme eingestellt hatte.

Es war wichtig, dass die Diagnose Delir gestellt wurde. Sie ist eine TriggerDiagnose, die den Einstieg in die weitere Abklärung eröffnet. Erst die Symptomkonstellation aus Ataxie, Schreien, Zittrigkeit und der motorischen Unru- he waren das Argument, die "demente Patientin mit Delir" in die Geriatrie zu verlegen. Wie sich rasch herausgestellt hat, ist die Patientin nicht dement gewesen. Sie hatte eine bis zur Aufnahme in der Klinik bestehende hohe Autonomie, relative Gesundheit und gute geistige Leistungsfähigkeit. Sie war anamnestisch depressiv und deswegen behandelt worden. Dabei ist es erstaunlich, dass die zuvor schon bestehende Kombinationstherapie aus Citalopram und Mirtazepin hinsichtlich der serotonergen Wirkung im Vorfeld bei dieser Patientin keine Nebenwirkungen gezeigt hatte.

Zwei Bereiche können die behandelnden Ärzte rasch auf die richtige Fährte des serotonergen Syndroms führen: 1. die Überprüfung von zusätzlichen neurologischen Symptomen: Die neurologischen Symptome mit Ataxie, Zittern, Erregbarkeit, Klonus, Hyperthermie, Tachykardie und Tachypnoe sind wegweisend für ein serotonerges Syndrom. 2. Die Überprüfung einer Risikokonstellation bei der Medikation: Die medikamentöse Vorbehandlung mit einem SSRi oder einem NaSSA stellt eine eindeutige Risikokonstellation für serotonerge Nebenwirkungen dar, insbesondere, wenn eine zusätzlich, evtl. rasch effektive Schmerzmedikation notwendig wird, wie in diesem Fall.

Möglicherweise war retrospektiv die $24 \mathrm{~h}$ nach Aufnahme dokumentierte delirante Symptomatik schon der Beginn des serotonergen Syndroms in sehr milder Form. Zu diesem Zeitpunkt hatte die
Patientin zusätzlich zu Citalopram und Mirtazapin das Schmerzmittel Oxycodon erhalten - auch diese Kombination ist in der Literatur als serotonerger "Mix" beschrieben [16].

Richtig entwickelt hat es sich retrospektiv aber erst unter der Pethidingabe mit einer Kumulation des Norpethidins unter kontinuierlicher i. v.-Gabe in sehr hohen Dosen. Auch dieser Mechanismus ist in der Literatur vorbeschrieben [27].

Die Wiederaufnahme der zentral wirkenden Medikamente muss sehr sorgfältig und kritisch gesehen werden, will man ein rasches Wiederaufflammen des serotonergen Syndroms vermeiden [28].

Fr. K. hatte mit ihren 89 Jahren ein mittelschweres bis schweres serotonerges Syndrom überlebt - dies war möglicherweise nur auf Grund der gesunden Ausgangslage und der Abwehrkraft der 89jährigen Frau möglich.

Der Zusammenhang der Verstoffwechslung der Medikamente über CYP3A4 und CYP2D6 zeigt in der pharmakologischen Bestimmung der Aktivität dieser beiden Zytochrome, wie wichtig für Alterspatienten eine solche Information vor Beginn von Therapien mit Medikamenten sein könnte. $\mathrm{Ob}$ eine Routinemessung der CYP-Aktivität bei Alterspatienten vielleicht sinnvoll wäre, könnte diskutiert werden.

In diesem Fall hatte das serotonerge Syndrom 9 Tage gedauert. Die Kosten eines serotonergen Syndroms sind von dem Zeitpunkt der Diagnose, der Bedingung und Aufrechterhaltung abhängig. Nimmt man die 9 Tage diesen Falls zur Grundlage, so wurden durch dieses serotonerge Syndrom für die Klinik $6400 €$ bzw. 19.200 sFr. an Kosten erzeugt. Dies kann zu einer kalkulatorischen Unterdeckung eines serotonergen Falls führen. Auch wenn das DRG-System keine direkte Abbildung von serotonergen Syndromen in der Matrix der Kostenabbildung vorhält, bietet es jedoch eine sehr wichtige Möglichkeit für die Versorgung dieser Patienten, nämlich die ökonomisch bewertete interdisziplinäre Zusammenarbeit von Abteilungen bei geriatrischen Patienten. Diese positiven Eigenschaften des DRGSystems müssten neben der qualitativen Versorgung auch hinsichtlich der ökonomischen Bewertung stärker im Wissen 
der behandelnden Ärzte verankert werden. Eine gezielte Frühsteuerung von solchen Patienten in eine kompetente Versorgung wie die Akutgeriatrie dürfte und sollte aus prognostischen und funktionellen Gründen angestrebt werden.

\section{Korrespondenzadresse}

\section{Dr. med. W. Weinrebe, MD, MSc}

University of Witten Herdecke

Witten-Herdecke, Germany

info@wep-medical.de

\section{Einhaltung ethischer Richtlinien}

Interessenkonflikt. W. Weinrebe, A. Moutaouakil, K. Risz, M. Martin, K. Jeckelmann und S. Goetz geben an, dass kein Interessenkonflikt besteht.

Dieser Beitrag beinhaltet keine von den Autoren durchgeführten Studien an Menschen oder Tieren. Alle Patienten, die über Bildmaterial oder anderweitige Angaben innerhalb des Manuskripts zu identifizieren sind, haben hierzu ihre schriftliche Einwilligung gegeben.

Open Access. Dieser Artikel wird unter der Creative Commons Namensnennung 4.0 International Lizenz (http://creativecommons.org/licenses/by/4.0/deed. de) veröffentlicht, welche die Nutzung, Vervielfältigung, Bearbeitung, Verbreitung und Wiedergabe in jeglichem Medium und Format erlaubt, sofern Sie den/die ursprünglichen Autor(en) und die Quelle ordnungsgemäßnennen, einen Link zur Creative Commons Lizenz beifügen und angeben, ob Änderungen vorgenommen wurden.

\section{Literatur}

1. Inouye SK, van Dyck CH, Alessi CA, Balkin S, Siegal AP, Horwitz RI (1990) Clarifying confusion: the confusion assessment method. A new method for detection of delirium. Ann Intern Med 113(12):941-948

2. Inouye SK, Kosar CM, Tommet D, Schmitt EM, Puelle MR, Saczynski JS, Marcantonio ER, Jones RN (2014) The CAM-S: development and validation of a new scoring system for delirium severity in 2 cohorts. Ann Intern Med 160(8):526-533. doi:10. 7326/m13-1927

3. Sternbach H (1991) The serotonin syndrome. Am J Psychiatry 148(6):705-713

4. Ener RA, Meglathery SB, Van Decker WA, Gallagher RM (2003) Serotonin syndrome and other serotonergic disorders. Pain Med 4(1):63-74

5. Jones D, Story DA (2005) Serotonin syndrome and the anaesthetist. Anaesth Intensive Care 33(2):181-187

6. Huang V, Gortney JS (2006) Risk of serotonin syndrome with concomitant administration of linezolid and serotonin agonists. Pharmacotherapy 26(12):1784-1793

7. Koury KM, Tsui B, Gulur P (2015) Incidence of serotonin syndrome in patients treated with fentanyl on serotonergic agents. Pain Physician 18:E27-E30

8. Ailawadhi S, Sung KW, Carlson LA, Baer MR (2007) Serotonin syndrome caused by interaction between citalopram and fentanyl. J Clin Pharm Ther 32(2):199-202. doi:10.1111/j.1365-2710. 2007.00813.x

9. Sato Y, Nakamura K, Yasui-Furukori N (2015) Serotonin syndrome induced by the readministration of escitalopram after a short-term interruption in an elderly woman with depression: a case report Neuropsychiatr Dis Treat 11:2505-2507

10. Dunkley EJ, Isbister GK, Sibbritt D, Dawson AH, Whyte IM (2003) The Hunter Serotonin Toxicity Criteria: simple and accurate diagnostic decision rules for serotonin toxicity. QJM 96(9):635-642

11. Ali SZ, Taguchi A, Rosenberg H (2003) Malignant hyperthermia. Best Pract Res Clin Anaesthesiol 17(4):519-533

12. Guzé BH, Baxter LR Jr. (1985) Current concepts. Neuroleptic malignant syndrome. N Engl J Med 313(3):163-166

13. von Bruckhausen F, EbelS, HackenthalE, Holzgrabe U (Hrsg) (1999) Handbuch der pharmazeutischen Praxis, Folgeband 5, Stoffe L-Z, 5. Aufl. Springer, Berlin Heidelberg, S247-248

14. www.pharmawiki.ch.Zugegriffen:27.Dez 2015

15. S 3 Leitlinie Behandlung akuter perioperativer und posttraumatischer Schmerzen, 2008, S. 55

16. Walter C, Ball D, Duffy M, Mellor JD (2012) An unusual case of serotonin syndrome with oxycodone and citalopram. Case Rep Oncol Med: doi:10.1155/2012/261787

17. Weinrebe W, Johannsdottir E, Karaman M, Füsgen I (2015) What does delirium cost? : An economic evaluation of hyperactive delirium. Z Gerontol Geriatr 49(1):52-58. doi:10.1007/s00391-0150871-6

18. Adrienne ZA, Raju N (2010) Prevention, diagnosis, and management of serotonin syndrome, residency program, Spartanburg, South Carolina. Am Fam Physician 81(9):1139-1142

19. Haberzettl R, Bert B, Fink H, Fox MA (2013) Animal models of the serotonin syndrome: a systematic review. Behav Brain Res 256:328-345. doi:10.1016/ j.bbr.2013.08.045

20. Lai MW, Klein-Schwartz W, Rodgers GC et al (2006) 2005 annual report of the American Association of Poison Control Centers' national poisoning and exopsure database. Clin Toxicol (Phila) 44(6-7):803-932. doi:10.1080/ 15563650600907165

21. Glaeske G, Schicktanz C et al (2012) Barmer GEK Arzneimittelreport 2012. Schriften zur Gesundheitsanalyse, Bd. 14.

22. Srisuma S, Hoyte C, Wongwisavakong S, Winauku W (2015) Serotoninsyndrom precipitated by sertraline. Clin Toxicol 43:840-841

23. Karunatilake H, Buckley NA (2006) Serotonin syndrome induced by fluvoxamine and oxycodone. Ann Pharmacother 40(1):155-157

24. Barmer GEK (2012) Arzneimittelreport 2012, Juni 2012. Schriften zur Gesundheitsanalyse, Bd. 14

25. Beakley BD, Kaye AM, Kaye AD (2015) Side effects, and serotonin syndrome: a review tramadol, pharmacology. Pain Physician 18:395-400

26. Martos V, Hofer KE, Rauber-Lüthy C, SchenkJaeger KM, Kupferschmidt H, Ceschi A (2015) Acute toxicity profile of tolperisone in overdose: observational poison centre-based study. Clin Toxicol (Phila) 53(5):470-476

27. Mateo-Carrasco H, Muñoz-Aguilera EM, GarcíaTorrecillas JM, Al-Robb AH (2015) Animal odel, serotonin syndrome probably triggered by a mor- phine-phenelzine interaction. Pharmacotherapy 35(6):e102-5.doi:10.1002/phar.1581

28. Sato Y, Nakamura K, Yasui-Furukori N (2015) Serotonin syndrome induced by the readministration of escitalopram after a short-term interruption in an elderly woman with depression: a case report. Neuropsychiatr Dis Treat 11:2505-2507 